Research Article

\title{
Novel Delay-Decomposing Approaches to Absolute Stability Criteria for Neutral-Type Lur'e Systems
}

\author{
Liang-Dong Guo $\stackrel{i D}{ }$, Sheng-Juan Huang, and Li-Bing Wu \\ School of Science, University of Science and Technology Liaoning, Anshan 114051, China \\ Correspondence should be addressed to Liang-Dong Guo; ldguo@ustl.edu.cn
}

Received 27 August 2019; Accepted 9 November 2019; Published 2 December 2019

Academic Editor: Mingshu Peng

Copyright (C) 2019 Liang-Dong Guo et al. This is an open access article distributed under the Creative Commons Attribution License, which permits unrestricted use, distribution, and reproduction in any medium, provided the original work is properly cited.

\begin{abstract}
The problem of absolute stability analysis for neutral-type Lur'e systems with time-varying delays is investigated. Novel delaydecomposing approaches are proposed to divide the variation interval of the delay into three unequal subintervals. Some new augment Lyapunov-Krasovskii functionals (LKFs) are defined on the obtained subintervals. The integral inequality method and the reciprocally convex technique are utilized to deal with the derivative of the LKFs. Several improved delay-dependent criteria are derived in terms of the linear matrix inequalities (LMIs). Compared with some previous criteria, the proposed ones give the results with less conservatism and lower numerical complexity. Two numerical examples are included to illustrate the effectiveness and the improvement of the proposed method.
\end{abstract}

\section{Introduction}

Over the last 30 years, time delay system has been one of the hottest research areas in control engineering for time delay often appears in many control systems either in the state, the control input, or the measurements $[1,2]$. Since time delay frequently occurs in practical systems and is often the source of instability, there have been many results for stability of delayed systems [3-9]. Also stabilization [10-12], filtering [13], and adaptive control [14] of time-delay systems have received considerable attention. The neutral systems often appear in the study of automatic control, population dynamics, and vibrating masses attached to an elastic bar [15]. A considerable number of studies related to this topic have been reported (see, for example, [16-21], and the references therein).

Lur'e system is originally from a pilot robot [22], which is one of the important classes of nonlinear systems whose nonlinear element satisfies certain sector constraints. Many systems such as Chua's circuits, Goodwin models, Swarm models, $n$-scroll attractors, and hyperchaotic attractors can be represented as Lur'e-type systems [23, 24]. In recent years, stability analysis of the neutral-type Lur'e system with time delays has attracted the attention of many researchers [25-35]. The main purpose of stability analysis is to calculate the maximum allowable delay bounds (MADBs), which is a key index for judging the conservatism of stability criteria, such that the Lur'e system maintains absolute stability for any time delay less than the MADBs by using the LKF method. In [25], absolute stability of Lur'e systems with sector-bounded nonlinearities and constant delay was discussed by using a Lur'e-Postnikov function. To avoid involving a considerable number of free-weighting matrices and leading to a computationally expensive stability criterion in [26], the general free weighting matrix method was proposed and some improved delay-range-dependent stability criteria were obtained [27, 28]. By using integral inequality and the Wirtinger integral inequality method instead of free weighting matrix one, some improved delaydependent robust stability criteria were derived in [29, 30], respectively. Also, by eliminating nonlinearity and reducing the number of free-weighting matrices, Lur'e systems with interval time-varying delays were discussed in [31]. By discretizing the delay interval into two segmentations with an unequal width, some delay-dependent sufficient conditions were given for the robust stability of neutral-type Lur'e systems in [32, 33]. By employing an augmented LKF method, reciprocally convex combination approach, and convex combination technique, several robust absolute 
stability criteria for uncertain neutral-type Lur'e systems with time-varying delays were presented in [34]. Very recently, by constructing a LKF including both double-integral terms and triple-integral terms, using the piecewise analysis method, Wirtinger-based integral inequality, and the reciprocally convex combination technique, some new stability criteria were obtained in [35]. In fact, one of the term $\int_{\left(t-\tau_{M}\right) / 2}^{t} \varrho_{1}^{T}(t) Q \varrho_{1}(t) \mathrm{d} s$ in LKFs in [35] is the special case of the delay-partitioning approach, where $\varrho_{1}(t)^{T}=\left[x^{T}(t)\right.$, $\left.x^{T}\left(\left(t-\tau_{M}\right) / 2\right)\right]^{T}$. The delay-partitioning method is an effective one to reduce a criterion's conservativeness, which is widely used in the stability analysis for various systems (see details in [36-39]). However, when utilizing the delaypartitioning approach, the term $\int_{t-\tau_{\mathrm{j}}}^{t} \varrho_{2}(t)^{T} Q \varrho_{2}(t) \mathrm{d} s$ is inevitably involved in LKFs, where $\varrho_{2}(t)^{T}=\left[x^{T}(t), x^{T}(t-\right.$ $\left.\left.\tau_{1}\right), \ldots, x^{T}\left(t-\tau_{N-1}\right)\right]^{T}, \tau_{i}=(d / N)$ and $N$ is the delaypartitioning number. It is clear that the derived conditions become more complicated and the computational burden grows bigger when $N$ increases. In addition, to relate to the Wirtinger-based integral inequality and deal with the derivative of triple-integral terms introduced in LKFs, it is ineluctable that the extra terms $\int_{\left(t-\tau_{M}\right) / 2}^{t} x^{T}(s) Q \mathrm{~d} s, \int_{t-\tau_{M}}^{\left(t-\tau_{M}\right) / 2}$ $x^{T}(s) Q \mathrm{~d} s, \int_{-\tau_{M} / 2}^{0} \int_{t+\theta}^{t} x^{T}(s) Q \mathrm{~d} s \mathrm{~d} \theta$, and $\int_{-\tau_{M}}^{-\tau_{M} / 2} \int_{t+\theta}^{t} x^{T}(s) Q$ $\mathrm{d} s \mathrm{~d} \theta$ had to be introduced into the derivation process in [35], which leads to a sharp increase in the dimensions of the LMIs involved.

Motivated by this mentioned above, the aim of this work is to revisit the stability analysis for the neutral-type Lur'e system. In this study, novel delay-decomposing approaches are proposed firstly. Different from the delay-partitioning approaches or the delay-decomposing ones in $[32,33$, $36-40]$, the interval of the state time delay $\left[0, \tau_{M}\right]$ is divided into three unequal subintervals $\left[0, \tau_{\alpha}\right],\left[\tau_{\alpha}, \tau_{\beta}\right]$, and $\left[\tau_{\beta}, \tau_{M}\right]$. In particular, to establish the relationship of the vectors such as $x(t), x\left(t-\tau_{\alpha}\right), x\left(t-\tau_{\beta}\right)$, and $x\left(t-\tau_{M}\right)$, the novel terms $\int_{t-\tau_{\alpha}}^{t} \varrho_{3}^{T}(t) Q \varrho_{3}(t) \mathrm{d} s$ and $\int_{t-\tau_{\beta}}^{t} \varrho_{4}^{T}(t) Q \varrho_{4}(t) \mathrm{d} s$ are introduced in LKFs in each of subintervals, which are more general than the ones in $[28,35]$, where $\varrho_{3}^{T}(t)=\left[x^{T}(t), x^{T}\left(t-\tau_{\beta}\right)\right]$ and $\varrho_{4}^{T}(t)=\left[x^{T}(t), x^{T}\left(t-\tau_{\alpha}\right)\right]$. It is worth mentioning that the merit of the proposed delay-decomposing method lies in that the dimensions of the LMIs involved are independent of the number of subinterval. In addition, to avoid introducing the extra vectors by Wirtinger-based integral inequality, the reciprocally convex combination method [41] and the integral inequality [42] are utilized to deal with the bounds of integral terms. Some novel LKFs related to the above inequalities are constructed on the obtained three subintervals. The presented stability criteria are given in terms of LMIs. Compared with the related literature, the conclusions of this paper have the advantages of less conservatism conservatism and the dimensions of the LMIs. Finally, two well-known numerical examples are given to demonstrate the effectiveness and less conservatism over the existing results.
Notation: in this paper, $\mathbb{R}^{n}$ denotes $n$-dimensional Euclidean space and $\mathbb{R}^{n \times m}$ is the set of all $n \times m$ real matrices. For symmetric matrices $X$ and $Y$, the notation $X>Y$ (respectively, $X \geq Y$ ) means that the matrix $X-Y$ is positive definite (respectively, nonnegative). diag $\{\cdots\}$ denotes the block diagonal matrix. The subscript " $T$ " denotes the transpose of the matrix. $I_{n}$ denotes the identity matrix.

\section{Problem Statements and Preliminaries}

Consider a class of Lur'e systems of neutral type with timevarying delays and sector-bound nonlinearities described as follows:

$$
\left\{\begin{array}{l}
\dot{x}(t)-C \dot{x}(t-d(t))=A x(t)+B x(t-\tau(t))+D f(\sigma(t)), \\
\sigma(t)=H^{T} x(t), \quad t \geq 0 \\
x(s)=\phi(s) \\
\dot{x}(s)=\dot{\phi}(s) \\
s \in\left[-\max \left(\tau_{M}, d_{M}\right), 0\right]
\end{array}\right.
$$

where $A, B, C$, and $D$ are constant matrices with appropriate dimensions. $x(t)=\left[x_{1}(t), x_{2}(t), \ldots, x_{n}(t)\right]^{T} \in \mathbb{R}^{n}$ is the state vector, and $\sigma(t)=\left[\sigma_{1}(t), \sigma_{2}(t), \ldots, \sigma_{m}(t)\right]^{T} \in \mathbb{R}^{m}$ is the output vector. The time delay $\tau(t)$ is a time-varying continuous function satisfying

$$
\begin{aligned}
0 & \leq \tau(t) \leq \tau_{M}, \\
\dot{\tau}(t) & \leq \mu_{\tau}, \\
0 & \leq d(t) \leq d_{M}, \\
0 & <\dot{d}(t) \leq \mu_{d},
\end{aligned}
$$

where $d_{M}, \tau_{M}, \mu_{\tau}$, and $\mu_{d}$ are the known constant scalars.

$f(\cdot)=\left[f_{1}(\cdot), f_{2}(\cdot), \ldots, f_{m}(\cdot)\right]^{T} \in \mathbb{R}^{m}$ is the nonlinear function and $f_{i}\left(\sigma_{i}(t)\right) \cdot(i=1,2, \ldots, m)$ is assumed to satisfy the finite sector restriction:

$$
\begin{aligned}
f_{i}\left(\sigma_{i}(t)\right) \in K_{\left[0, k_{i}\right]} & =\left\{f_{i}\left(\sigma_{i}(t)\right) \mid f_{i}(0)=0,0<\sigma(t) f_{i}\left(\sigma_{i}(t)\right)\right. \\
& \left.\leq k_{i} \sigma^{2}(t), \sigma_{i}(t) \neq 0\right\},
\end{aligned}
$$

with known positive scalar $k_{i}$ or the infinite sector restriction:

$$
\begin{aligned}
f_{i}\left(\sigma_{i}(t)\right) \in K_{[0, \infty]} & =\left\{f_{i}\left(\sigma_{i}(t)\right) \mid f_{i}(0)\right. \\
& \left.=0, \sigma(t) f_{i}\left(\sigma_{i}(t)\right)>0, \sigma_{i}(t) \neq 0\right\} .
\end{aligned}
$$

The objective of this paper is to formulate the delaydependent stability conditions of system (1). The following lemmas will play important roles in deriving the criteria.

Lemma 1 (see [41]). Let $f_{1}, f_{2}, \ldots, f_{N}: \mathbb{R}^{m} \longmapsto \mathbb{R}$ have positive values in an open subset $D$ of $\mathbb{R}^{m}$. Then, the reciprocally convex combination of $f_{i}$ over $D$ satisfies 


$$
\min _{\left\{\alpha_{i} \mid \alpha_{i}>0, \sum_{i} \alpha_{i}=1\right\}} \sum_{i} \frac{1}{\alpha_{i}} f_{i}(t)=\sum_{i} f_{i}(t)+\max _{g_{i, j}(t)} \sum_{i \neq j} g_{i, j}(t),
$$

subject to

$$
\left\{g_{i, j}: R^{m} \longrightarrow R, g_{j, i}(t)=g_{i, j}(t),\left[\begin{array}{cc}
f_{i}(t) & g_{i, j}(t) \\
g_{j, i}(t) & f_{j}(t)
\end{array}\right] \geq 0\right\} .
$$

Lemma 2 (see [42]). For any matrices $Q>0, M, N, X$ with compatible dimensions, any continuous time vector function $x(t)$ and $\eta(t)$ with compatible dimensions, and any scalar $\tau_{M}$ satisfying $0 \leq \tau(t) \leq \tau_{M}$, the following integral inequality holds:

$$
\begin{aligned}
-\int_{t-\tau_{M}}^{t} x^{\mathrm{T}}(s) Q x(s) \mathrm{d} s \leq & \tau_{M} \eta^{\mathrm{T}}(t) X \eta(t)+2 \eta^{\mathrm{T}}(t) \\
& \cdot\left\{M \int_{t-\tau_{M}}^{t-\tau(t)} x(s) \mathrm{d} s+N \int_{t-\tau(t)}^{t} x(s) \mathrm{d} s\right\},
\end{aligned}
$$

if $\left[\begin{array}{cc}Q & M^{T} \\ M & X\end{array}\right] \geq 0$ and $\left[\begin{array}{cc}Q & N^{T} \\ N & X\end{array}\right] \geq 0$.

Remark 1. Let $M=N$ and $\eta^{T}(t)=\left[x^{T}(t), x^{T}(t-\tau(t))\right.$, $x^{T}\left(t-\tau_{M}\right)$ ], the integral inequality reduces the one in [43].

\section{Main Result}

In this section, some new delay-dependent stability criteria are proposed for system (1).

Note $\tau_{\alpha}=\alpha \tau_{M}$ and $\tau_{\beta}=\beta \tau_{M}(0 \leq \alpha \leq 0.5, \beta+\alpha=1)$. It is easy to see that $0 \leq \tau_{\alpha} \leq \tau_{\beta} \leq \tau_{M}$ holds. Then, we divide the time interval $\left[0, \tau_{M}\right]$ into three subintervals $\Delta_{1}=:\left[0, \tau_{\alpha}\right]$, $\Delta_{2}=:\left[\tau_{\alpha}, \tau_{\beta}\right]$, and $\Delta_{3}=:\left[\tau_{\beta}, \tau_{M}\right]$. In the following, we will propose some criteria for the three subintervals.

Now, we give the stability criteria for system (1) with conditions (2) and (4) when $\tau(t) \in \Delta_{1}$ as follows.

Theorem 1. For given scalars $0 \leq \alpha \leq 0.5, \tau_{M} \geq 0,0 \leq \mu_{d}<1$, $\mu_{\tau} \geq 0$, and $k_{i}>0,(i=1,2, \ldots, m)$, system (1) with conditions (2) and (4) is absolutely stable if there exist $n \times n$ symmetric positive definite matrices $P, S_{1}, S_{2}, R_{1}, R_{2}, R_{3}, 2 n \times 2 n$ symmetric positive definite matrices $Q_{1}, Q_{2}, 2 n \times 2 n$ symmetric semi-positive definite matrices $Y_{11}, Z_{11}$, positive diagonal matrices $U=\operatorname{diag}\left\{u_{1}, u_{2}, \ldots, u_{m}\right\}, L=\operatorname{diag}\left\{l_{1}, l_{2}, \ldots, l_{m}\right\}$, any $n \times n$ matrix $X_{12}$, and $2 n \times n$ matrices $Y_{12}, Z_{12}$, such that LMIs (5) and (6) hold when $\tau(t) \in \Delta_{1}$ :

$$
\begin{aligned}
& \Xi_{1}+\Xi_{1}^{T}+\Xi_{2}+\Xi_{3}+\Xi_{4}+\Xi_{4}^{T}<0 \\
& \Phi_{i} \geq 0, \quad i=1,2,3
\end{aligned}
$$

where

$$
\begin{aligned}
& \Phi_{1}=\left[\begin{array}{cc}
R_{1} & X_{12} \\
X_{12}^{T} & R_{1}
\end{array}\right], \\
& \Phi_{2}=\left[\begin{array}{cc}
Y_{11} & Y_{12} \\
Y_{12}^{T} & R_{2}
\end{array}\right] \text {, } \\
& \Phi_{3}=\left[\begin{array}{cc}
Z_{11} & Z_{12} \\
Z_{12}^{T} & R_{3}
\end{array}\right] \text {, } \\
& \Xi_{1}=\Xi_{10}+\left[e_{3}, e_{4}\right] Y_{12}\left(e_{3}^{T}-e_{4}^{T}\right)+\left(\left[e_{4}, e_{5}\right] Z_{12}\left(e_{4}^{T}-e_{5}^{T}\right)\right)^{T} \text {, } \\
& \Xi_{10}=e_{1} P e_{8}^{T}+e_{7} L H^{T} e_{8}^{T}, \\
& \Xi_{20}=e_{1} S_{1} e_{1}^{T}-\left(1-\mu_{\tau}\right) e_{2} S_{1} e_{2}^{T}-\left(1-\mu_{d}\right) e_{6} S_{2} e_{6}^{T} \\
& +\left[e_{1}, e_{4}\right] Q_{1}\left[e_{1}, e_{4}\right]^{T}-\left[e_{3}, e_{5}\right] Q_{1}\left[e_{3}, e_{5}\right]^{T} \\
& +\left[e_{1}, e_{3}\right] Q_{2}\left[e_{1}, e_{3}\right]^{T}-\left[e_{4}, e_{5}\right] Q_{2}\left[e_{4}, e_{5}\right]^{T} \text {, } \\
& \Xi_{2}=\Xi_{20}-2 e_{7} U e_{7}^{T} \text {, } \\
& \Xi_{3}=e_{8}\left[S_{2}+\tau_{\alpha}^{2} R_{1}+\left(\tau_{\beta}-\tau_{\alpha}\right) R_{2}+\left(\tau_{M}-\tau_{\beta}\right) R_{3}\right] e_{8}^{T} \\
& -\left[e_{2}-e_{1}, e_{3}-e_{2}\right] \Phi_{1}\left[e_{2}-e_{1}, e_{3}-e_{2}\right]^{T} \\
& +\left(\tau_{\beta}-\tau_{\alpha}\right)\left[e_{3}, e_{4}\right] Y_{11}\left[e_{3}, e_{4}\right]^{T}+\left(\tau_{M}-\tau_{\beta}\right) \\
& \cdot\left[e_{4}, e_{5}\right] Z_{11}\left[e_{4}, e_{5}\right]^{T} \text {, } \\
& \Xi_{4}=e_{1} \mathrm{HKU}_{7}^{T} \text {, } \\
& e_{8}=e_{1} A^{T}+e_{5} B^{T}+e_{6} C^{T}+e_{7} D^{T}, \\
& K=\operatorname{diag}\left\{k_{1}, k_{2}, \ldots, k_{m}\right\} \text {, } \\
& e_{1}=\left[I_{n}, 0_{n \times n}, 0_{n \times n}, 0_{n \times n}, 0_{n \times n}, 0_{n \times m}\right]^{T}, \\
& e_{2}=\left[0_{n \times n}, I_{n}, 0_{n \times n}, 0_{n \times n}, 0_{n \times n}, 0_{n \times m}\right]^{T}, \\
& e_{3}=\left[0_{n \times n}, 0_{n \times n}, I_{n}, 0_{n \times n}, 0_{n \times n}, 0_{n \times m}\right]^{T}, \\
& e_{4}=\left[0_{n \times n}, 0_{n \times n}, 0_{n \times n}, 0_{n \times n}, I_{n}, 0_{n \times m}\right]^{T}, \\
& e_{5}=\left[0_{n \times n}, 0_{n \times n}, 0_{n \times n}, 0_{n \times n}, I_{n}, 0_{n \times m}\right]^{T} \text {, } \\
& e_{6}=\left[0_{n \times n}, 0_{n \times n}, 0_{n \times n}, 0_{n \times n}, 0_{n \times m}, I_{n}\right]^{T} \text {, } \\
& e_{7}=\left[0_{m \times n}, 0_{m \times n}, 0_{m \times n}, 0_{m \times n}, 0_{m \times n}, I_{m}\right]^{T} \text {. }
\end{aligned}
$$

Proof. For positive diagonal matrices $L=\operatorname{diag}\left\{l_{1}, l_{2}, \ldots, l_{m}\right\}$ and positive definite matrices $P, R_{j}, S_{i}$, and $Q_{i} \cdot(i=1,2$, $j=1,2,3)$, let us consider the following LKF candidates for the case $0 \leq \tau(t) \leq \tau_{\alpha}$ :

$$
V(x(t))=V_{1}(x(t))+V_{2}(x(t))+V_{3}(x(t)),
$$

where 


$$
\begin{aligned}
V_{1}(x(t))= & x^{T}(t) P x(t)+\int_{t-\tau(t)}^{t} x^{T}(s) S_{1} x(s) \mathrm{d} s \\
& \left.+\int_{t-d(t)}^{t} \dot{x}^{T}(s) S_{2} \dot{x}(s) \mathrm{d} s+2 \sum_{i=1}^{m} l_{i}\right]_{0}^{\sigma_{i}} f_{i}(\sigma) \mathrm{d} \sigma, \\
V_{2}(x(t))= & \int_{t-\tau_{\alpha}}^{t}\left[\begin{array}{c}
x(s) \\
x\left(s-\tau_{\beta}\right)
\end{array}\right]^{T} Q_{1}\left[\begin{array}{c}
x(s) \\
x\left(s-\tau_{\beta}\right)
\end{array}\right] \mathrm{d} s \\
& +\int_{t-\tau_{\beta}}^{t}\left[\begin{array}{c}
x(s) \\
x\left(s-\tau_{\alpha}\right)
\end{array}\right]^{T} Q_{2}[x(s) \\
V_{3}(x(t))= & \tau_{\alpha} V_{31}(x(t))+V_{32}(x(t))+V_{33}(x(t)), \\
V_{31}(x(t))= & \int_{-\tau_{a}}^{0} \int_{t+\theta}^{t} \dot{x}^{T}(s) R_{1} \dot{x}(s) \mathrm{d} s \mathrm{~d} \theta, \\
V_{32}(x(t))= & \int_{-\tau_{\beta}}^{-\tau_{\alpha}} \int_{t+\theta}^{t} \dot{x}^{T}(s) R_{2} \dot{x}(s) \mathrm{d} s \mathrm{~d} \theta, \\
V_{33}(x(t))= & \int_{-\tau_{M}}^{-\tau_{\alpha}} \int_{t+\theta}^{t} \dot{x}^{T}(s) R_{3} \dot{x}(s) \mathrm{d} s \mathrm{~d} \theta .
\end{aligned}
$$

The time derivative of $V(x(t))$ along the trajectory of system (4) is given by

$$
\begin{aligned}
\dot{V}_{1}(x(t)) \leq & 2 x^{T}(t) P \dot{x}(t)+2 f^{T}(x(t)) \mathrm{LH}^{T} \dot{x}(t)+x^{T}(t) S_{1} x(t) \\
& +\dot{x}^{T}(t) S_{2} \dot{x}(t)-\left(1-\mu_{\tau}\right) x(t-\tau(t))^{T}(t) \\
& \cdot S_{1} x(t-\tau(t))-\left(1-\mu_{d}\right) \dot{x}(t-d(t))^{T}(t) S_{2} \dot{x}(t-d(t)) \\
= & \xi^{T}(t)\left[e_{1} P e_{8}^{T}+e_{8} P e_{1}^{T}+e_{1} S_{1} e_{1}^{T}-\left(1-\mu_{\tau}\right) e_{2} S_{1} e_{2}^{T}\right. \\
& \left.-\left(1-\mu_{d}\right) e_{6} S_{2} e_{6}^{T}+e_{7} L H^{T} e_{8}^{T}+e_{8} H L^{T} e_{7}^{T}+e_{8} S_{2} e_{8}^{T}\right] \xi(t),
\end{aligned}
$$

where

$$
\begin{aligned}
\xi^{T}(t)= & {\left[x^{T}(t), x^{T}(t-\tau(t)), x^{T}\left(t-\tau_{\alpha}\right), x^{T}\left(t-\tau_{\beta}\right),\right.} \\
& \left.x^{T}\left(t-\tau_{M}\right), \dot{x}^{T}(t-d(t)), f^{T}(\sigma(t))\right],
\end{aligned}
$$

$$
\begin{aligned}
\dot{V}_{2}(x(t))= & {\left[\begin{array}{c}
x(t) \\
x\left(t-\tau_{\beta}\right)
\end{array}\right]^{T} Q_{1}\left[\begin{array}{c}
x(t) \\
x\left(t-\tau_{\beta}\right)
\end{array}\right] } \\
& -\left[\begin{array}{c}
x\left(t-\tau_{\alpha}\right) \\
x\left(t-\tau_{M}\right)
\end{array}\right]^{T} Q_{1}\left[\begin{array}{c}
x\left(t-\tau_{\alpha}\right) \\
x\left(s-\tau_{M}\right)
\end{array}\right] \\
& +\left[\begin{array}{c}
x(t) \\
x\left(t-\tau_{\alpha}\right)
\end{array}\right]^{T} Q_{2}\left[\begin{array}{c}
x(t) \\
x\left(t-\tau_{\alpha}\right)
\end{array}\right] \\
& -\left[\begin{array}{c}
x\left(t-\tau_{\beta}\right) \\
x\left(t-\tau_{M}\right)
\end{array}\right]^{T} Q_{2}\left[\begin{array}{c}
x\left(t-\tau_{\beta}\right) \\
x\left(s-\tau_{M}\right)
\end{array}\right] \\
= & \xi^{T}(t)\left\{\left[e_{1}, e_{4}\right] Q_{1}\left[e_{1}, e_{4}\right]^{T}-\left[e_{3}, e_{5}\right] Q_{1}\left[e_{3}, e_{5}\right]^{T}\right. \\
& \left.+\left[e_{1}, e_{3}\right] Q_{2}\left[e_{1}, e_{3}\right]^{T}-\left[e_{4}, e_{5}\right] Q_{2}\left[e_{4}, e_{5}\right]^{T} \xi(t)\right\},
\end{aligned}
$$

$$
\begin{aligned}
\dot{V}_{3}(x(t))= & \dot{x}^{T}(t)\left[\tau_{\alpha}^{2} R_{1}+\left(\tau_{\beta}-\tau_{\alpha}\right) R_{2}+\left(\tau_{M}-\tau_{\beta}\right) R_{3}\right] \dot{x}(t) \\
& -\tau_{\alpha} \int_{t-\tau_{\alpha}}^{t} \dot{x}^{T}(s) R_{1} \dot{x}(s) \mathrm{d} s-\int_{t-\tau_{\beta}}^{t-\tau_{\alpha}} \dot{x}^{T}(s) R_{2} \dot{x}(s) \mathrm{d} s \\
& -\int_{t-\tau_{M}}^{t-\tau_{\beta}} \dot{x}^{T}(s) R_{3} \dot{x}(s) \mathrm{d} s .
\end{aligned}
$$

If $0 \leq \tau(t) \leq \tau_{\alpha}$ holds, one can compute out the following according to Lemma 1:

$$
\begin{aligned}
& -\tau_{\alpha} \int_{t-\tau_{\alpha}}^{t} \dot{x}^{T}(s) R_{1} \dot{x}(s) \mathrm{d} s \\
& \quad=-\tau_{\alpha} \int_{t-\tau(t)}^{t} \dot{x}^{T}(s) R_{1} \dot{x}(s) \mathrm{d} s-\tau_{\alpha} \int_{t-\tau_{\alpha}}^{t-\tau(t)} \dot{x}^{T}(s) R_{1} \dot{x}(s) \mathrm{d} s \\
& \quad \leq-\left[\begin{array}{c}
x(t-\tau(t))-x(t) \\
x\left(t-\tau_{\alpha}\right)-x(t-\tau(t))
\end{array}\right]^{T} \Phi_{1}\left[\begin{array}{c}
x(t-\tau(t))-x(t) \\
x\left(t-\tau_{\alpha}\right)-x(t-\tau(t))
\end{array}\right] \\
& =-\xi^{T}(t)\left[e_{2}-e_{1}, e_{3}-e_{2}\right] \Phi_{1}\left[e_{2}-e_{1}, e_{3}-e_{2}\right]^{T} \xi(t),
\end{aligned}
$$

where $\Phi_{1}$ is given in (6).

Based on Lemma 2 (in which $M=N=Y_{12}$ and $\left.\eta^{T}(t)=\left[x^{T}\left(t-\tau_{\alpha}\right), x^{T}\left(t-\tau_{\beta}\right)\right]\right)$, when $\Phi_{2}=\left[\begin{array}{cc}Y_{11} & Y_{12} \\ Y_{12}^{t} & R_{2}\end{array}\right] \geq$ 0 , the following inequality holds:

$$
\begin{aligned}
& -\int_{t-\tau_{\beta}}^{t-\tau_{\alpha}} \dot{x}^{T}(s) R_{2} \dot{x}(s) \mathrm{d} s \\
& \leq\left(\tau_{\beta}-\tau_{\alpha}\right)\left[\begin{array}{c}
x\left(t-\tau_{\alpha}\right) \\
x\left(t-\tau_{\beta}\right)
\end{array}\right]^{T} Y_{11}\left[\begin{array}{l}
x\left(t-\tau_{\alpha}\right) \\
x\left(t-\tau_{\beta}\right)
\end{array}\right] \\
& \quad+2\left[\begin{array}{l}
x\left(t-\tau_{\alpha}\right) \\
x\left(t-\tau_{\beta}\right)
\end{array}\right]^{T} Y_{12}\left[x\left(t-\tau_{\alpha}\right)-x\left(t-\tau_{\beta}\right)\right] \\
& =\xi^{T}(t)\left\{\left(\tau_{\beta}-\tau_{\alpha}\right)\left[e_{3}, e_{4}\right] Y_{11}\left[e_{3}, e_{4}\right]^{T}+\left[e_{3}, e_{4}\right]\right. \\
& \left.\quad \cdot Y_{12}\left(e_{3}^{T}-e_{4}^{T}\right)+\left(\left[e_{3}, e_{4}\right] Y_{12}\left(e_{3}^{T}-e_{4}^{T}\right)\right)^{T}\right\} \xi(t),
\end{aligned}
$$

and using Lemma 2 again (in which $M=N=Z_{12}$ and $\left.\eta^{T}(t)=\left[x^{T}\left(t-\tau_{\beta}\right), x^{T}\left(t-\tau_{M}\right)\right]\right)$, when $\Phi_{3}=\left[\begin{array}{cc}Z_{11} & Z_{12} \\ Z_{12}^{t} & R_{3}\end{array}\right]$ $\geq 0$, one can conclude

$$
\begin{aligned}
- & \int_{t-\tau_{M}}^{t-\tau_{\beta}} \dot{x}^{T}(s) R_{3} \dot{x}(s) \mathrm{d} s \\
\leq & \left(\tau_{M}-\tau_{\beta}\right)\left[\begin{array}{c}
x\left(t-\tau_{\beta}\right) \\
x\left(t-\tau_{M}\right)
\end{array}\right]^{T} Z_{11}\left[\begin{array}{c}
x\left(t-\tau_{\beta}\right) \\
x\left(t-\tau_{M}\right)
\end{array}\right] \\
& +2\left[\begin{array}{c}
x\left(t-\tau_{\beta}\right) \\
x\left(t-\tau_{M}\right)
\end{array}\right]^{T} Z_{12}\left[x\left(t-\tau_{\beta}\right)-x\left(t-\tau_{M}\right)\right] \\
= & \xi^{T}(t)\left\{\left(\tau_{M}-\tau_{\beta}\right)\left[e_{4}, e_{5}\right] Z_{11}\left[e_{4}, e_{5}\right]^{T}\right. \\
& \left.+\left[e_{4}, e_{5}\right] Z_{12}\left(e_{4}^{T}-e_{5}^{T}\right)+\left(\left[e_{4}, e_{5}\right] Z_{12}\left(e_{4}^{T}-e_{5}^{T}\right)\right)^{T}\right\} \xi(t) .
\end{aligned}
$$


Under the assumption on nonlinear function (4), the following inequality holds

$$
u_{i} f_{i}(\sigma(t))\left[k_{i} h_{i}^{T} x(t)-f_{i}\left(\sigma_{i}(t)\right)\right] \geq 0, \quad i=1,2, \ldots, m
$$

It is equivalent to

$$
2\left[x^{T}(t) \operatorname{HKU} f(\sigma(t))-f^{T}(\sigma(t)) U f(\sigma(t))\right] \geq 0 .
$$

Rewrite the above as follows:

$$
2\left[e_{1} \mathrm{HKU} e_{7}^{T}-e_{7} U e_{7}^{T}\right] \geq 0,
$$

where

$$
\begin{aligned}
& U=\operatorname{diag}\left\{u_{1}, u_{2}, \ldots, u_{m}\right\}, \\
& K=\operatorname{diag}\left\{k_{1}, k_{2}, \ldots, k_{m}\right\} .
\end{aligned}
$$

Then, combining (3)-(15) leads to

$$
\dot{V}(x(t)) \leq \xi^{T}(t)\left(\Xi_{1}+\Xi_{1}^{T}+\Xi_{2}+\Xi_{3}+\Xi_{4}+\Xi_{4}^{T}\right) \xi(t) .
$$

Therefore, if LMIs (5) and (6) hold, one has $\dot{V}(x(t))<0$, which shows the absolute stability of system (1) subject to (2) and (4), when $\tau(t)$ satisfies $C_{1}$. This completes our proof.

For the case $\tau(t) \in \Delta_{2}$, we construct the LKFs as follows:

$$
\bar{V}(x(t))=V_{1}(x(t))+V_{2}(x(t))+\bar{V}_{3}(x(t)),
$$

where

$$
\bar{V}_{3}(x(t))=V_{31}(x(t))+\left(\tau_{\beta}-\tau_{\alpha}\right) V_{32}(x(t))+V_{33}(x(t)),
$$

and $V_{1}(x(t)), V_{2}(x(t)), V_{31}(x(t))$, and $V_{33}(x(t))$ are defined in (7).

Then, the derivative of $\bar{V}_{3}(x(t))$ can be obtained as

$$
\begin{aligned}
\overline{V_{3}}(x(t))= & \dot{x}^{T}(t)\left[\tau_{\alpha} R_{1}+\left(\tau_{\beta}-\tau_{\alpha}\right)^{2} R_{2}+\left(\tau_{M}-\tau_{\beta}\right) R_{3}\right] \dot{x}(t) \\
& -\int_{t-\tau_{\alpha}}^{t} \dot{x}^{T}(s) R_{1} \dot{x}(s) \mathrm{d} s-\left(\tau_{\beta}-\tau_{\alpha}\right) \int_{t-\tau_{\beta}}^{t-\tau_{\alpha}} \dot{x}^{T}(s) R_{2} \dot{x}(s) \mathrm{d} s \\
& -\int_{t-\tau_{M}}^{t-\tau_{\beta}} \dot{x}^{T}(s) R_{3} \dot{x}(s) \mathrm{d} s .
\end{aligned}
$$

According to Lemma 1 , if $\tau_{\alpha} \leq \tau(t) \leq \tau_{\beta}$, the following inequality holds:

$$
\begin{aligned}
-\left(\tau_{\beta}-\tau_{\alpha}\right) \int_{t-\tau_{\beta}}^{t-\tau_{\alpha}} \dot{x}^{T}(s) R_{2} \dot{x}(s) \mathrm{d} s \\
=-\left(\tau_{\beta}-\tau_{\alpha}\right) \int_{t-\tau(t)}^{t-\tau_{\alpha}} \dot{x}^{T}(s) R_{2} \dot{x}(s) \mathrm{d} s, \\
-\left(\tau_{\beta}-\tau_{\alpha}\right) \int_{t-\tau_{\beta}}^{t-\tau(t)} \dot{x}^{T}(s) R_{2} \dot{x}(s) \mathrm{d} s \\
\leq-\left[\begin{array}{l}
x(t-\tau(t))-x\left(t-\tau_{\alpha}\right) \\
x\left(t-\tau_{\beta}\right)-x(t-\tau(t))
\end{array}\right]^{T} \\
\quad . \Phi_{2}\left[\begin{array}{l}
x(t-\tau(t))-x\left(t-\tau_{\alpha}\right) \\
x\left(t-\tau_{\beta}\right)-x(t-\tau(t))
\end{array}\right] \\
=-\xi^{T}(t)\left[e_{2}-e_{3}, e_{4}-e_{2}\right] \bar{\Phi}_{2}\left[e_{2}-e_{3}, e_{4}-e_{2}\right]^{T} \xi(t),
\end{aligned}
$$

where $\bar{\Phi}_{2}$ is given in (20).

In addition, by using Lemma 2 (in which $M=N=X_{12}$ and $\left.\eta^{T}(t)=\left[x^{T}(t), x^{T}\left(t-\tau_{\alpha}\right)\right]\right)$, if $\bar{\Phi}_{1}=\left[\begin{array}{cc}X_{11} & X_{12}^{t} \\ X_{12} & R_{1}\end{array}\right] \geq 0$, one can obtain

$$
\begin{aligned}
& -\int_{t-\tau_{\alpha}}^{t} \dot{x}^{T}(s) R_{1} \dot{x}(s) \mathrm{d} s \\
& \leq \tau_{\alpha}\left[\begin{array}{c}
x(t) \\
x\left(t-\tau_{\alpha}\right)
\end{array}\right]^{T} X_{11}\left[\begin{array}{c}
x(t) \\
x\left(t-\tau_{\alpha}\right)
\end{array}\right] \\
& \quad+2\left[\begin{array}{c}
x(t) \\
x\left(t-\tau_{\alpha}\right)
\end{array}\right]^{T} X_{12}\left[x(t)-x\left(t-\tau_{\alpha}\right)\right] \\
& =\xi^{T}(t)\left\{\tau_{\alpha}\left[e_{1}, e_{3}\right] X_{11}\left[e_{1}, e_{3}\right]^{T}+\left[e_{1}, e_{3}\right] X_{12}\left(e_{1}^{T}-e_{3}^{T}\right)\right. \\
& \left.\quad+\left(\left[e_{1}, e_{3}\right] X_{12}\left(e_{1}^{T}-e_{3}^{T}\right)\right)^{T}\right\} \xi(t) .
\end{aligned}
$$

The other procedure is straightforward from the proof of Theorem 1; we can cope with the situation of $\tau(t) \in \Delta_{2}$ and have the following.

Theorem 2. For given scalars $0 \leq \alpha \leq 0.5, \tau_{M} \geq 0,0 \leq \mu_{d}<1$, $\mu_{\tau} \geq 0$, and $k_{i}>0(i=1,2, \ldots, m)$, system (1) with conditions (2) and (4) is absolutely stable if there exist $n \times n$ symmetric positive definite matrices $P, S_{1}, S_{2}, R_{1}, R_{2}, R_{3}, 2 n \times 2 n$ symmetric positive definite matrices $Q_{1}, Q_{2}, 2 n \times 2 n$ symmetric semi-positive definite matrices $X_{11}, Z_{11}$, positive diagonal matrices $U=\operatorname{diag}\left\{u_{1}, u_{2}, \ldots, u_{m}\right\}, L=\operatorname{diag}\left\{l_{1}, l_{2}, \ldots, l_{m}\right\}$, any $n \times n$ matrix $Y_{12}$, and $2 n \times n$ matrices $X_{12}, Z_{12}$, such that LMIs (19) and (20) hold for the case $\tau(t) \in \Delta_{2}$ :

$$
\bar{\Xi}_{1}+\bar{\Xi}_{1}^{T}+\Xi_{2}+\bar{\Xi}_{3}+\Xi_{4}+\Xi_{4}^{T}<0 \text {, }
$$

$$
\begin{aligned}
& \bar{\Phi}_{1} \geq 0 \\
& \bar{\Phi}_{2} \geq 0 \\
& \Phi_{3} \geq 0
\end{aligned}
$$

where

$$
\begin{aligned}
\bar{\Phi}_{1}= & {\left[\begin{array}{cc}
X_{11} & X_{12} \\
X_{12}^{t} & R_{1}
\end{array}\right], } \\
\bar{\Phi}_{2}= & {\left[\begin{array}{cc}
R_{2} & Y_{12} \\
Y_{12}^{t} & R_{2}
\end{array}\right], } \\
\bar{\Xi}_{1}= & \Xi_{10}+\left[e_{1}, e_{3}\right] X_{12}\left(e_{1}^{T}-e_{3}^{T}\right) \\
& +\left[e_{4}, e_{5}\right] Z_{12}\left(e_{4}^{T}-e_{5}^{T}\right), \\
\bar{\Xi}_{3}= & e_{8}\left[S_{2}+\tau_{\alpha} R_{1}+\left(\tau_{\beta}-\tau_{\alpha}\right)^{2} R_{2}+\left(\tau_{M}-\tau_{\beta}\right) R_{3}\right] e_{8}^{T} \\
& -\left[e_{2}-e_{3}, e_{4}-e_{2}\right] \bar{\Phi}_{2}\left[e_{2}-e_{3}, e_{4}-e_{2}\right]^{T} \\
& +\tau_{\alpha}\left[e_{1}, e_{3}\right] X_{11}\left[e_{1}, e_{3}\right]^{T} \\
& +\left(\tau_{M}-\tau_{\beta}\right)\left[e_{4}, e_{5}\right] Z_{11}\left[e_{4}, e_{5}\right]^{T}
\end{aligned}
$$

and $\Phi_{3}, \Gamma_{10}, \Gamma_{2}$, and $\Gamma_{4}\left(e_{i}, i=1,2, \ldots, 8\right)$ and $K$ are defined in Theorem 1 . 
For the case $\tau(t) \in \Delta_{3}$, we construct the following LKF:

$$
\widehat{V}(x(t))=V_{1}(x(t))+V_{2}(x(t))+\widehat{V}_{3}(x(t)),
$$

where

$$
\widehat{V}_{3}(x(t))=V_{31}(x(t))+V_{32}(x(t))+\left(\tau_{M}-\tau_{\beta}\right) V_{33}(x(t))
$$

and $V_{1}(x(t)), V_{2}(t), V_{31}(t)$, and $V_{32}(t)$ are defined in (7).

Taking the derivative of $\widehat{V}_{3}(x(t))$ yields

$$
\begin{aligned}
\dot{\hat{V}}_{3}(x(t))= & \dot{x}^{T}(t)\left[\tau_{\alpha} R_{1}+\left(\tau_{\beta}-\tau_{\alpha}\right) R_{2}+\left(\tau_{M}-\tau_{\beta}\right)^{2} R_{3}\right] \dot{x}(t) \\
& -\int_{t-\tau_{\alpha}}^{t} \dot{x}^{T}(s) R_{1} \dot{x}(s) \mathrm{d} s \\
& -\int_{t-\tau_{\beta}}^{t-\tau_{\alpha}} \dot{x}^{T}(s) R_{2} \dot{x}(s) \mathrm{d} s-\left(\tau_{M}-\tau_{\beta}\right) \\
& \cdot \int_{t-\tau_{M}}^{t-\tau_{\beta}} \dot{x}^{T}(s) R_{3} \dot{x}(s) \mathrm{d} s .
\end{aligned}
$$

By using Lemma 1, if $\tau_{\beta} \leq \tau(t) \leq \tau_{M}$, one can obtain

$$
\begin{aligned}
-\left(\tau_{M}-\tau_{\beta}\right) \int_{t-\tau_{M}}^{t-\tau_{\beta}} \dot{x}^{T}(s) R_{3} \dot{x}(s) \mathrm{d} s \\
=-\left(\tau_{M}-\tau_{\beta}\right) \int_{t-\tau(t)}^{t-\tau_{\beta}} \dot{x}^{T}(s) R_{3} \dot{x}(s) \mathrm{d} s-\left(\tau_{\beta}-\tau_{\alpha}\right) \\
\cdot \int_{t-\tau_{M}}^{t-\tau(t)} \dot{x}^{T}(s) R_{3} \dot{x}(s) \mathrm{d} s \\
\leq- \\
\quad\left[\begin{array}{c}
x(t-\tau(t))-x\left(t-\tau_{\beta}\right) \\
x\left(t-\tau_{M}\right)-x(t-\tau(t))
\end{array}\right]^{T} \widehat{\Phi}_{3} \\
\cdot\left[\begin{array}{c}
x(t-\tau(t))-x\left(t-\tau_{\beta}\right) \\
x\left(t-\tau_{M}\right)-x(t-\tau(t))
\end{array}\right] \\
=-\xi^{T}(t)\left[e_{2}-e_{4}, e_{5}-e_{2}\right] \widehat{\Phi}_{3}\left[e_{2}-e_{4}, e_{5}-e_{2}\right]^{T} \xi(t),
\end{aligned}
$$

where $\widehat{\Phi}_{3}=\left[\begin{array}{cc}R_{3} & Z_{12}^{t} \\ Z_{12} & R_{3}\end{array}\right]$.

Handle the integral terms $-\int_{t-\tau_{\alpha}}^{t} \dot{x}^{T}(s) R_{1} \dot{x}(s) d s$ and $-\int_{t-\tau_{\beta}}^{t-\tau_{\alpha}} \dot{x}^{T}(s) R_{2} \dot{x}(s) d s$ in the same way as in (13) and (17), respectively. Following the same procedure, we can cope with the situation of $\tau(t) \in \Delta_{3}$ and have the following.

Theorem 3. For given scalars $0 \leq \alpha \leq 0.5, \tau_{M} \geq 0,0 \leq \mu_{d}<1$, $\mu_{\tau} \geq 0$, and $k_{i}>0(i=1,2, \ldots, m)$, system (1) with conditions (2) and (4) is absolutely stable if there exist $n \times n$ symmetric positive definite matrices $P, S_{1}, S_{2}, R_{1}, R_{2}, R_{3}, 2 n \times 2 n$ symmetric positive definite matrices $Q_{1}, Q_{2}, 2 n \times 2 n$ symmetric semi-positive definite matrices $X_{11}, Y_{11}$, positive diagonal matrices $U=\operatorname{diag}\left\{u_{1}, u_{2}, \ldots, u_{m}\right\}, L=\operatorname{diag}\left\{l_{1}, l_{2}, \ldots, l_{m}\right\}$, any $n \times n$ matrices $Z_{12}$, and $2 n \times n$ matrices $X_{12}, Y_{12}$, such that LMIs (23) and (24) hold for the case $\tau(t) \in \Delta_{3}$ :

$$
\widehat{\Xi}_{1}+\widehat{\Xi}_{1}^{T}+\Xi_{2}+\widehat{\Xi}_{3}+\Xi_{4}+\Xi_{4}^{T}<0
$$

$$
\begin{aligned}
& \bar{\Phi}_{1} \geq 0 \\
& \Phi_{2} \geq 0 \\
& \widehat{\Phi}_{3} \geq 0
\end{aligned}
$$

where

$$
\begin{aligned}
\widehat{\Phi}_{3}= & {\left[\begin{array}{cc}
R_{3} & Z_{12} \\
Z_{12}^{t} & R_{3}
\end{array}\right], } \\
\widehat{\Xi}_{3}= & e_{8}\left[S_{2}+\tau_{\alpha} R_{1}+\left(\tau_{\beta}-\tau_{\alpha}\right) R_{2}+\left(\tau_{M}-\tau_{\beta}\right)^{2} R_{3}\right] e_{8}^{T} \\
& +\tau_{\alpha}\left[e_{1}, e_{3}\right] X_{11}\left[e_{1}, e_{3}\right]^{T} \\
& +\left(\tau_{\beta}-\tau_{\alpha}\right)\left[e_{3}, e_{4}\right] Y_{11}\left[e_{3}, e_{4}\right]^{T}, \\
\widehat{\Xi}_{1}= & \Xi_{10}+\left[e_{1}, e_{3}\right] X_{12}\left(e_{1}^{T}-e_{3}^{T}\right) \\
& +\left[e_{3}, e_{4}\right] Y_{12}\left(e_{3}^{T}-e_{4}^{T}\right),
\end{aligned}
$$

and $\Phi_{2}, \Gamma_{10}, \Gamma_{2}, \Gamma_{4}, e_{i}(i=1,2, \ldots, 8), K$, and $\bar{\Phi}_{1}$ are defined in Theorems 1 and 2, respectively.

Remark 2. Theorems 1-3 present the absolute stability criteria for the Lur'e system. Unlike the delay-partitioning approach or delay-decomposing one used in $[28,32,33$, $35,40]$, the interval of the state time delay $\left[0, \tau_{M}\right]$ has been divided into three subintervals $\left[0, \tau_{\alpha}\right],\left[\tau_{\alpha}, \tau_{\beta}\right]$, and $\left[\tau_{\beta}, \tau_{M}\right]$. Obviously, the range of the subintervals $\left[0, \tau_{\alpha}\right],\left[\tau_{\alpha}, \tau_{\beta}\right]$, and $\left[\tau_{\beta}, \tau_{M}\right]$ is $\alpha \tau_{M},(1-2 \alpha) \tau_{M}$, and $\alpha \tau_{M}$. Thus, to know more about the time-varying $\tau(t)$, one can select the value of the parameter $\alpha$ as close as possible to 0 for the case $\tau(t) \in \Delta_{1}$ or case $\tau(t) \in \Delta_{3}$ and as close as possible to 0.5 for the case $\tau(t) \in \Delta_{2}$, respectively. The following examples in the work will illustrate the point.

Remark 3. The terms $\int_{t-\tau_{\alpha}}^{t} \varrho_{3}^{T}(t) Q_{1} \varrho_{3}(t) \mathrm{d} s$ and $\int_{t-\tau_{\beta}}^{t} \varrho_{4}^{T}(t)$ $Q_{2} \varrho_{4}(t) \mathrm{d} s$ have been introduced in LKFs in the work instead of the terms $\int_{t-\tau_{M} / 2}^{t} \varrho_{1}^{T}(t) Q \varrho_{1}(t) \mathrm{d} s$ in $[28,35]$ and $\int_{t-\tau_{i}}^{t} \varrho_{2}$ $(t)^{T} \mathrm{Q} \varrho_{2}(t) \mathrm{d} s$ in [36-39], where $\varrho_{1}(t)^{T}=\left[x^{T}(t), x^{T}((t-\right.$ $\left.\left.\left.\tau_{M}\right) / 2\right)\right]^{T}$ and $\varrho_{2}(t)^{T}=\left[x^{T}(t), x^{T}\left(t-\tau_{1}\right), \ldots, x^{T}\left(t-\tau_{N-1}\right)\right]^{T}$. And the relations between $x(t), x\left(t-\tau_{\alpha}\right), x\left(t-\tau_{\beta}\right)$, and $x\left(t-\tau_{M}\right)$ have been effectively expressed by the derivative of $V_{2}(x(t))$. One can easily see that $\int_{t-\tau_{\alpha}}^{t} \varrho_{3}^{T}(t) Q_{1} \varrho_{3}(t) \mathrm{d} s$ or $\int_{t-\tau_{\beta}}^{t} \varrho_{4}^{T}(t) Q_{2} \varrho_{4}(t) \mathrm{d} s$ deduces into $\int_{t-\tau_{M} / 2}^{t} \varrho_{1}^{T}(t) Q \varrho_{1}(t) \mathrm{d} s$ when $\alpha=\beta=0.5$. This is to say the established LKFs are more general than ones in $[28,35]$. The idea is expected to reduce the conservatism of obtained criteria. On the other 
hand, it can effectively overcome the weakness of the computational burden growing bigger when delay-partitioning number increases in [36-39].

Remark 4. In [35], the Wirtinger-based integral inequality and double-integral inequality were used to deal with bounds of the derivative of double-integral and triple-integral terms in LKFs. Thus, the extra terms $\int_{t-\tau_{M} / 2}^{t}$ $x^{T}(s) Q \mathrm{~d} s, \int_{t-\tau_{M}}^{t-\tau_{M} / 2} x^{T}(s) Q \mathrm{~d} s, \int_{-\tau_{M} / 2}^{0} \int_{t+\theta}^{t} x^{T}(s) Q \mathrm{~d} s \mathrm{~d} \theta$, and $\int_{-\tau_{M}}^{-\tau_{M} / 2} \int_{t+\theta}^{t} x^{T}(s) Q \mathrm{~d} s \mathrm{~d} \theta$ were unavoidably introduced in the derivation process, which leads the dimensions of the LMIs involved to increase sharply. A detailed comparison is given in Example 1.

Remark 5. If function $f(\sigma(t))$ of system (1) satisfies sector condition (3), for any $u_{i} \geq 0, i=1,2, \ldots, m$, one has the following:

$$
u_{i} f_{i}\left(\sigma_{i}(t)\right) h_{i}^{T} x(t) \geq 0, \quad i=1,2, \ldots, m,
$$

which is equivalent to

$$
\begin{array}{r}
2 x^{T}(t) \operatorname{HU} f(\sigma(t)) \geq 0 \\
\text { or } 2 \xi^{T}(t) e_{1} \mathrm{HU}_{7}^{T} \xi(t) \geq 0 .
\end{array}
$$

Thus, if function $f(\sigma(t))$ of system (1) satisfies sector condition (3), we have the following corollary.

Corollary 1. For given scalars $0 \leq \alpha \leq 0.5, \tau_{M} \geq 0,0 \leq \mu_{d}<1$, $\mu_{\tau} \geq 0, k_{i}>0, i=1,2, \ldots, m$, the system (1) with conditions (2) and (3) is absolutely stable if there exist symmetric positive definite matrices $P, S_{1}, S_{2}, R_{1}, R_{2}, R_{3}, Q_{1}, Q_{2}, X_{11}, Y_{11}$ with appropriate dimensions, positive diagonal matrices $U=\operatorname{diag}$ $\left\{u_{1}, u_{2}, \ldots, u_{m}\right\}, L=\operatorname{diag}\left\{l_{1}, l_{2}, \ldots, l_{m}\right\}$, and matrices $X_{11}$, $Y_{11}, Z_{11}, X_{12}, Y_{12}, Z_{12}$ with appropriate dimensions and properties, such that LMIs (6) and (26) hold for the case $\tau(t) \in \Delta_{1}$, (3) and (28) hold for the case $\tau(t) \in \Delta_{2}$, and (20) and (28) hold for the case $\tau(t) \in \Delta_{3}$, respectively:

$$
\begin{aligned}
& \Xi_{1}+\Xi_{1}^{T}+\Xi_{20}+\Xi_{3}+\Xi_{40}+\Xi_{40}^{t}<0, \\
& \bar{\Xi}_{1}+\bar{\Xi}_{1}^{T}+\Xi_{20}+\bar{\Xi}_{3}+\Xi_{40}+\Xi_{40}^{t}<0, \\
& \widehat{\Xi}_{1}+\widehat{\Xi}_{1}^{T}+\Xi_{20}+\widehat{\Xi}_{3}+\Xi_{40}+\Xi_{40}^{t}<0,
\end{aligned}
$$

where $\Gamma_{40}=e_{1} H U e_{7}^{T}, \Gamma_{1}, \bar{\Gamma}_{1}, \widehat{\Gamma}_{1}, \Gamma_{20}, \Gamma_{3}, \bar{\Gamma}_{3}, \widehat{\Gamma}_{3}$ are defined in Theorems 1-3.

\section{Illustrative Example}

In this section, we will use two well-known numerical examples to show the effectiveness and benefits of our results.
Example 1. Consider the following nominal neutral-type Lur'e system (1) subject to (2) and (4) with the parameters:

$$
\begin{aligned}
& A=\left[\begin{array}{cc}
-2 & 0.5 \\
0 & -1
\end{array}\right], \\
& B=\left[\begin{array}{cc}
1 & 0.4 \\
0.4 & -1
\end{array}\right], \\
& C=\left[\begin{array}{ll}
0.2 & 0.1 \\
0.1 & 0.2
\end{array}\right], \\
& D=\left[\begin{array}{l}
-0.5 \\
-0.75
\end{array}\right], \\
& H=\left[\begin{array}{l}
0.2 \\
0.6
\end{array}\right] .
\end{aligned}
$$

The purpose of this example is to compute MADBs of $\tau_{M}$ such that the neutral-type Lur'e system (1) remains stable for different $\mu_{\tau}$ and $\mu_{d}$. For given $\mu_{d}=0.9, \alpha=0.45$, $\mu_{d}=0.5, \alpha=0.45$, and $\mu_{d}=0.1, \alpha=0.4$, the acceptable upper bounds of $\tau_{M}$ is $0.271,2.277$, and 2.652 when $\mu_{\tau} \geq 1$ by using Corollary $1\left(\tau(t) \in \Delta_{1}\right)$ in our work, respectively. In order to make a comparison with some existing stability criteria, we calculate the MADBs and list them in Tables 1-3. Together with all derived MADBs listed in Tables 1-3, one can check that Corollary 1 can be superior over some present ones. The number of decision variables, maximal order of LMIs between our work, and the criteria in $[29,30,33,35]$ are listed in Table 4 . It shows that our proposed method involves smaller decision variables or lower maximal order of LMIs than the relative ones. To confirm the obtained result, a simulation result is shown in Figure 1 when $\mu$ is unknown, $x(0)=[0,0.4]^{T}, \tau_{M}=5.886$, and $h(t)=0.5+0.1 \sin t$.

Example 2. Consider Chua's circuit example discussed in [35]:

$$
\left\{\begin{array}{l}
\dot{\theta}_{1}(t)=a\left(\theta_{2}-g\left(\theta_{1}\right)\right), \\
\dot{\theta}_{2}(t)=\theta_{1}-\theta_{2}+\theta_{3}, \\
\dot{\theta}_{3}(t)=-b \theta_{2} .
\end{array}\right.
$$

The nonlinear function $g\left(\theta_{1}\right)=m_{1} \theta_{1}+0.5\left(m_{0}-m_{1}\right)$ $\left(\left|\theta_{1}+c\right|-\left|\theta_{1}-c\right|\right)$. Let $m_{0}=-1 / 7, m_{1}=2 / 7, a=9, b=$ 14.28, and $c=1$; then, Chua's circuit can be expressed as a Lur'e-type system with 
TABLE 1: MADBs of $\tau_{M}$ for different $\mu_{\tau}$, when $\mu_{d}=0.1$ for Example 1.

\begin{tabular}{lcccc}
\hline Method & $\mu_{\tau}=0.2$ & $\mu_{\tau}=0.4$ & $\mu_{\tau}=0.6$ & $\mu_{\tau}=0.8$ \\
\hline$[44]$ & 2.6052 & 1.9538 & 1.6232 & 1.1659 \\
{$[31]$} & 2.6062 & 1.9730 & 1.6408 & 1.1854 \\
{$[27]$} & 2.6488 & 2.0056 & 1.6679 & 1.2049 \\
{$[30]$} & 2.8890 & 2.0678 & 1.7048 & 1.6059 \\
{$[32]$} & 2.9962 & 2.1316 & 1.7138 & 1.3204 \\
{$[28]$} & 2.9997 & 2.1012 & 1.7307 & 1.3359 \\
{$[29]$} & 3.0717 & 2.1171 & 1.7310 & 1.3367 \\
{$[33]$} & 3.1034 & 2.1579 & 1.7436 & 1.7088 \\
{$[34]$} & 3.1743 & 2.1789 & 1.7467 & 1.7153 \\
{$[35]$} & 3.4880 & 2.3787 & 1.8062 & 1.4625 \\
Cor $1\left(\tau(t) \in \Delta_{1}, \alpha=0.45\right)$ & 5.886 & 4.456 & 3.705 & 2.676 \\
Cor 1 $\left(\tau(t) \in \Delta_{2}, \alpha=0.3\right)$ & 3.784 & 2.865 & 2.385 & 2.176 \\
Cor 1 $\left(\tau(t) \in \Delta_{3}, \alpha=0.4\right)$ & 3.060 & 2.157 & 2.000 & 1.999 \\
Cor $1\left(\tau(t) \in \Delta_{3}, \alpha=0.04\right)$ & 3.729 & 3.729 & 3.729 & 3.729 \\
\hline
\end{tabular}

TABLE 2: MADBs of $\tau_{M}$ for different $\mu_{\tau}$, when $\mu_{d}=0.5$ for Example 1.

\begin{tabular}{lcccc}
\hline Method & $\mu_{\tau}=0.2$ & $\mu_{\tau}=0.4$ & $\mu_{\tau}=0.6$ & $\mu_{\tau}=0.8$ \\
\hline$[29]$ & 2.5131 & 1.8146 & 1.4951 & 1.1168 \\
{$[44]$} & 2.1532 & 1.6986 & 1.3687 & 1.0066 \\
{$[31]$} & 2.1537 & 1.7041 & 1.3828 & 1.0095 \\
{$[27]$} & 2.1883 & 1.7322 & 1.4056 & 1.0262 \\
{$[30]$} & 2.3628 & 1.7752 & 1.5027 & 1.4364 \\
{$[32]$} & 2.4472 & 1.8289 & 1.4551 & 1.1058 \\
{$[28]$} & 2.4563 & 1.8009 & 1.4823 & 1.1157 \\
{$[33]$} & 2.5302 & 1.8461 & 1.5371 & 1.5208 \\
{$[35]$} & 2.8538 & 2.0236 & 1.5679 & 1.2425 \\
Cor $1\left(\tau(t) \in \Delta_{1}, \alpha=0.45\right)$ & 4.862 & 3.848 & 3.119 & 2.280 \\
Cor $1\left(\tau(t) \in \Delta_{2}, \alpha=0.3\right)$ & 3.126 & 2.474 & 2.101 & 1.922 \\
Cor $1\left(\tau(t) \in \Delta_{3}, \alpha=0.04\right)$ & 2.877 & 2.877 & 2.877 & 2.877 \\
Cor $1\left(\tau(t) \in \Delta_{3}, \alpha=0.4\right)$ & 2.504 & 1.847 & 1.738 & 1.737 \\
\hline
\end{tabular}

TABLE 3: MADBs of $\tau_{M}$ for different $\mu_{\tau}$, when $\mu_{d}=0.9$ for Example 1 .

\begin{tabular}{lcccc}
\hline Method & $\mu_{\tau}=0.2$ & $\mu_{\tau}=0.4$ & $\mu_{\tau}=0.6$ & $\mu_{\tau}=0.8$ \\
\hline$[31]$ & 0.1122 & 0.1088 & 0.1086 & 0.1086 \\
{$[27]$} & 0.1122 & 0.1089 & 0.1086 & 0.1086 \\
{$[32]$} & 0.1126 & 0.1102 & 0.1102 & 0.1102 \\
{$[28]$} & 0.1130 & 0.1105 & 0.1105 & 0.1105 \\
{$[44]$} & 0.1221 & 0.1087 & 0.1083 & 0.1083 \\
{$[29]$} & 0.1227 & 0.1197 & 0.1197 & 0.1197 \\
{$[35]$} & 0.1799 & 0.1697 & 0.1660 & 0.1658 \\
{$[30]$} & 0.2495 & 0.2366 & 0.2300 & 0.2285 \\
{$[33]$} & 0.2906 & 0.2745 & 0.2653 & 0.2621 \\
Cor 1 $\left(\tau(t) \in \Delta_{1}, \alpha=0.35\right)$ & 0.321 & 0.311 & 0.310 & 0.310 \\
Cor 1 $\left(\tau(t) \in \Delta_{1}, \alpha=0.4\right)$ & 0.281 & 0.272 & 0.271 & 0.271 \\
Cor 1 $\left(\tau(t) \in \Delta_{2}, \alpha=0.4\right)$ & 0.189 & 0.189 & 0.189 & 0.189 \\
Cor 1 $\left(\tau(t) \in \Delta_{3}, \alpha=0.4\right)$ & 0.113 & 0.113 & 0.113 & 0.113 \\
Cor 1 $\left(\tau(t) \in \Delta_{3}, \alpha=0.04\right)$ & 0.119 & 0.119 & 0.119 & 0.119 \\
\hline
\end{tabular}

TABLE 4: Number of decision variables (NDV) and maximal order of LMIs (MOL) of different methods.

\begin{tabular}{lcc}
\hline Method & NDV & MOL \\
\hline [29] Theorem 1 & $12 n^{2}+7 n+2 m$ & $7 n+m$ \\
[35] Theorem 10 & $19.5 n^{2}+6.5 n+2 m$ & $10 n+m$ \\
[30] Theorem 1 & $11.5 n^{2}+7 n+2 m$ & $10 n+m$ \\
[33] Theorem 1 & $20 n^{2}+6 n+2 m$ & $12 n+m$ \\
Our Cor 1 & $16 n^{2}+7 n+2 m$ & $6 n+m$ \\
\hline
\end{tabular}

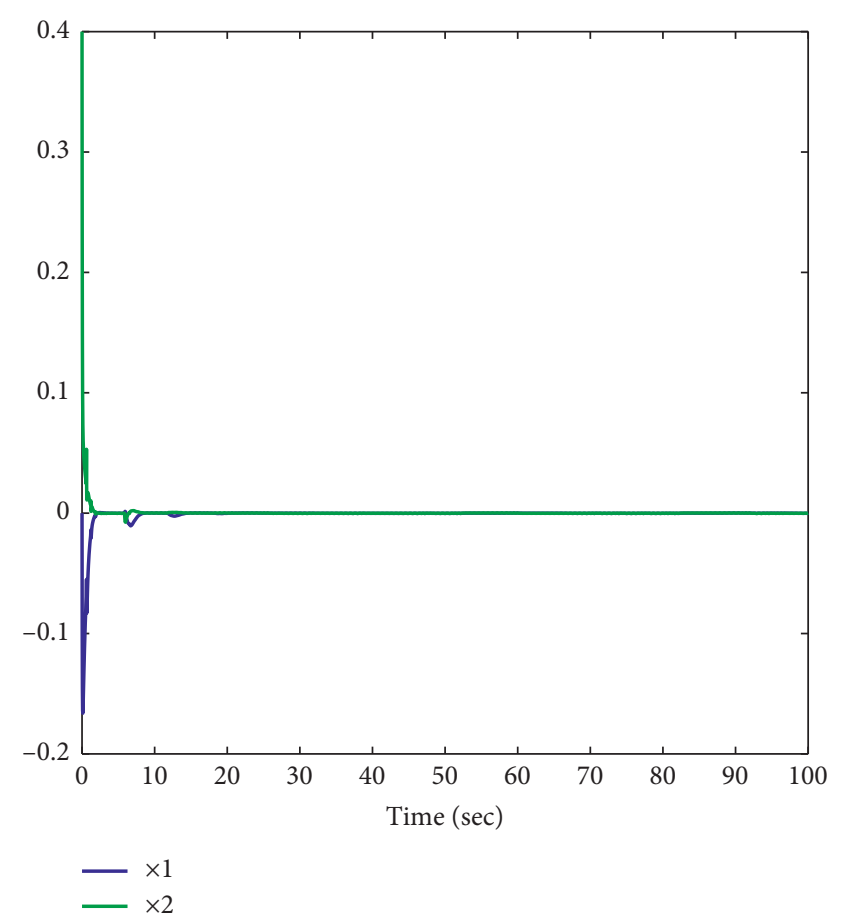

FIgURE 1: State responses of the system considered in Example 1.

$$
\begin{aligned}
A & =\left[\begin{array}{ccc}
-a m_{1}-1 & a & 0 \\
1 & -2 & 1 \\
0 & -b & -1
\end{array}\right], \\
B & =\left[\begin{array}{ccc}
-6.0029 & 0 & 0 \\
-1.3367 & 0 & 0 \\
2.1264 & 0 & 0
\end{array}\right], \\
C & =\left[\begin{array}{lll}
0 & 0 & 0 \\
0 & 0 & 0 \\
0 & 0 & 0
\end{array}\right], \\
D & =\left[\begin{array}{cc}
-a\left(m_{0}-m_{1}\right) \\
0
\end{array}\right], \\
H & =\left[\begin{array}{ll}
1 \\
0 \\
0
\end{array}\right] .
\end{aligned}
$$

The feedback nonlinear function belongs to $K_{[0,1]}$.

Now, we calculate MADBs of $\tau_{M}$. For different $\mu_{\tau}$, the obtained results are given in Table 5 . From this table, it is clear to see that Theorems 1 and 2 offer larger MADBs of $\tau_{M}$ than those methods in existing references.

\section{Conclusions}

This paper has investigated the absolute stability analysis for neutral-type Lur'e systems with time-varying delays. Based on the new delay-decomposition approaches in combination 
TABLe 5: MADBs $\tau_{M}$ for different $\mu_{\tau}$, when $\mu_{d}=0.1$ for Example 2 .

\begin{tabular}{lccccc}
\hline Method & $\mu_{\tau}=0$ & $\mu_{\tau}=0.3$ & $\mu_{\tau}=0.6$ & $\mu_{\tau}=0.9$ & $>1$ \\
\hline$[24]$ & 0.1622 & 0.1591 & 0.1566 & 0.1541 & 0.1527 \\
{$[27]$} & 0.1745 & 0.1698 & 0.1698 & 0.1698 & 0.1698 \\
{$[29]$} & 0.1747 & 0.1710 & 0.1703 & 0.1703 & 0.1703 \\
{$[32]$} & 0.1771 & 0.1721 & 0.1715 & 0.1715 & 0.1715 \\
{$[35]$} & 0.2790 & 0.2691 & 0.2553 & 0.2463 & 0.2463 \\
$\begin{array}{l}\text { Theorem 1 } \\
(\alpha=0.45)\end{array}$ & 0.361 & 0.359 & 0.359 & 0.359 & 0.359 \\
$\begin{array}{l}\text { Theorem 2 } \\
(\alpha=0.45)\end{array}$ & 0.295 & 0.295 & 0.295 & 0.295 & 0.295 \\
$\begin{array}{l}\text { Theorem 3 } \\
(\alpha=0.01)\end{array}$ & 0.163 & 0.163 & 0.163 & 0.163 & 0.163 \\
\hline
\end{tabular}

with the integral inequality and reciprocally convex technique, several improved stability criteria have been derived by constructing some appropriate LKFs on the subintervals. The merit of the obtained stability criteria lies in the significant less conservativeness and lower computational complexity than some existing ones. Finally, two examples have been given to demonstrate the effectiveness and less conservatism of the proposed method. In the future works, we will be dedicated to study the stability analysis for systems with infinite delays and devote to the study of output feedback, tracking control and filtering of the neutral-type Lur'e systems with time-varying delays based on the method proposed in this paper.

\section{Data Availability}

All data generated or analyzed during this study are included in this article.

\section{Conflicts of Interest}

The authors declare that they have no conflicts of interest.

\section{Acknowledgments}

This work was supported by the National Natural Science Foundation of China under Grants 61503058 and 61773013.

\section{References}

[1] J.-K. Hale and S.-M. Lunel, Introduction to Functional Differential Equations, Springer-Verlag, Berlin, Germany, 1993.

[2] J.-H. Park, H. Lee, Y. Liu, and J. Chen, Dynamic Systems with Time Delays: Stability and Control, Springer-Nature, Berlin, Germany, 2019.

[3] O.-M. Kwon, J.-H. Park, and S.-M. Lee, "Augmented Lyapunov functional approach to stability of uncertain neutral systems with time-varying delays," Applied Mathematics and Computation, vol. 207, no. 1, pp. 202-212, 2009.

[4] X.-M. Zhang, Q.-L. Han, A. Seuret, and F. Gouaisbaut, "An improved reciprocally convex inequality and an augmented Lyapunov-Krasovskii functional for stability of linear systems with time-varying delay," Automatica, vol. 84, pp. 221-226, 2017.

[5] C.-K. Zhang, Y. He, L. Jiang, M. Wu, and Q.-G. Wang, "An extended reciprocally convex matrix inequality for stability analysis of systems with time-varying delay," Automatica, vol. 85, pp. 481-485, 2017.

[6] J.-H. Park and S. Won, "A note on stability of neutral delaydifferential systems," Journal of the Franklin Institute, vol. 336, no. 3, pp. 543-548, 1999.

[7] E. Fridman, "New Lyapunov-Krasovskii functionals for stability of linear retarded and neutral type systems," Systems \& Control Letters, vol. 43, no. 4, pp. 309-319, 2001.

[8] K. Liu, E. Fridman, K. H. Johansson, and Y. Xia, "Generalized Jensen inequalities with application to stability analysis of systems with distributed delays over infinite time-horizons," Automatica, vol. 69, pp. 222-231, 2016.

[9] K. Liu, A. Seuret, Y. Xia, F. Gouaisbaut, and Y. Ariba, "BesselLaguerre inequality and its application to systems with infinite distributed delays," Automatica, vol. 109, pp. 1-7, 2019.

[10] C. Hua, S. Wu, and X. Guan, "Stabilization of T-S fuzzy system with time delay under sampled-data control using a new looped-functional," IEEE Transactions on Fuzzy Systems, p. 1, 2019.

[11] G.-Zhang, J.-Xia, W.-H. Zhang, J.-S. Zhao, Q. Sun, and H.-S. Zhang, "State feedback control for stochastic Markovian jump delay systems based on LaSalle-type theorem," Journal of the Franklin Institute, vol. 335, no. 5, pp. 2179-2196, 2018.

[12] G.-M. Zhang, J.-W. Xia, and W.-H. Zhang, "Normalization design for delayed singular Markovian jump systems based on system transformation technique," International Journal of Systems Science, vol. 49, no. 8, pp. 1603-1614, 2018.

[13] G.-M. Zhuang, S.-Y. Xu, B.-Y. Zhang, H.-L. Xu, and Y. Chu, "RobustHoodeconvolution filtering for uncertain singular Markovian jump systems with time-varying delays," International Journal of Robust and Nonlinear Control, vol. 26, no. 12, pp. 2564-2585, 2016.

[14] C.-C. Hua, G.-P. Liu, L. Li, and X.-P. Guan, “Adaptive fuzzy prescribed performance control for nonlinear switched timedelay systems with unmodeled dynamics," IEEE Transactions on Fuzzy Systems, vol. 26, no. 4, pp. 1934-1945, 2018.

[15] D. Yue and Q.-L. Han, "A delay-dependent stability criterion of neutral systems and its application to a partial element equivalent circuit model," IEEE Transactions on Circuits and Systems, vol. 6, no. 12, pp. 5438-5442, 2004.

[16] Q.-L. Han, “A descriptor system approach to robust stability of uncertain neutral systems with discrete and distributed delays," Automatica, vol. 40, no. 10, pp. 1791-1796, 2004.

[17] Y. He, M. Wu, J.-H. She, and G.-P. Liu, "Delay-dependent robust stability criteria for uncertain neutral systems with mixed delays," Systems \& Control Letters, vol. 51, no. 1, pp. 57-65, 2004.

[18] O.-M. Kwon, J.-H. Park, and S.-M. Lee, "On delay-dependent robust stability of uncertain neutral systems with interval time-varying delays," Applied Mathematics and Computation, vol. 203, no. 2, pp. 843-853, 2008.

[19] L.-D. Guo, H. Gu, and D.-Q. Zhang, "Robust stability criteria for uncertain neutral system with interval time varying discrete delay," Asian Journal of Control, vol. 12, no. 6, pp. 739-745, 2010.

[20] G.-M. Zhuang, S.-Y. Xu, J.-W. Xia, Q. Ma, and Z.-Q. Zhang, "Non-fragile delay feedback control for neutral stochastic Markovian jump systems with time-varying delays," Applied Mathematics and Computation, vol. 355, pp. 21-32, 2019.

[21] G.-M. Zhuang, J.-W. Xia, J.-E. Feng, B.-Y. Zhang, J.-W. Lu, and Z. Wang, "Admissibility analysis and stabilization for neutral descriptor hybrid systems with time-varying delays," Nonlinear Analysis: Hybrid Systems, vol. 33, pp. 311-321, 2019.

[22] A. I. Lur'e, Some Nonlinear Problems in the Theory of Automatic Control, H. M. Stationery Office, London, UK, 1957. 
[23] M. E. Yal'in, J. A. K. Suykens, and J. Vandewalle, "Masterslave synchronization of Lure systems with time-delay," International Journal of Bifurcation and Chaos, vol. 11, no. 6, pp. 1707-1722, 2001.

[24] C.-Y. Yang, Q.-L. Zhang, J. Sun, and T.-Y. Chai, "Lur'e Lyapunov function and absolute stability criterion for Lur'e singularly perturbed systems," IEEE Transactions on Automatic Control, vol. 56, no. 11, pp. 2666-2671, 2011.

[25] X. Liu, J.-Z. Wang, Z.-S. Duan, and L. Huang, "New absolute stability criteria for time-delay Lur'e systems with sectorbounded nonlinearity," International Journal of Robust Nonlinear Control, vol. 20, no. 6, pp. 659-672, 2010.

[26] C. Yin, S.-M. Zhong, and W.-F. Chen, "On delay-dependent robust stability of a class of uncertain mixed neutral and Lur'e dynamical systems with interval time-varying delays," Journal of the Franklin Institute, vol. 27, no. 5, pp. 515-516, 2010.

[27] K. Ramakrishnan and G. Ray, "Improved delay-range-dependent robust stability criteria for a class of Lur'e systems with sector-bounded nonlinearity," Journal of the Franklin Institute, vol. 348, no. 8, pp. 1769-1786, 2011.

[28] K. Ramakrishnan and G. Ray, "An improved delay-dependent stability criterion for a class of Lur'e systems of neutral type," Siam Journal on Applied Dynamical Systems, vol. 134, no. 1, pp. 1-6, 2012.

[29] W.-Y. Duan, B.-Z. Du, Z.-F. Liu, and Y. Zou, "Improved stability criteria for uncertain neutral-type Lur'e systems with time-varying delays," Journal of the Franklin Institute, vol. 351, no. 9, pp. 4538-4554, 2014.

[30] Y.-J. Liu, S.-M. Lee, O.-M. Kwon, and J.-H. Park, "Robust delay-dependent stability criteria for time-varying delayed Lur'e systems of neutral type," Circuits, Systems, and Signal Processing, vol. 34, no. 5, pp. 1481-1497, 2015.

[31] Y.-T. Wang, X. Zhang, and Y. He, "Improved delay-dependent robust stability criteria for a class of uncertain mixed neutral and Lur'e dynamical systems with interval timevarying delays and sector-bounded nonlinearity," Nonlinear Analysis: Real World Applications, vol. 13, no. 5, pp. 21882194, 2012.

[32] W.-Y. Duan, B.-Z. Du, J. You, and Y. Zou, "Improved robust stability criteria for a class of Lur'e systems with interval timevarying delays and sector-bounded nonlinearity," International Journal of Systems Science, vol. 46, no. 5, pp. 944-954, 2015.

[33] W.-Y. Duan, X.-R. Fu, Z.-F. Liu, and X.-D. Yang, "Improved robust stability criteria for time-delay Lur'e system," Asian Journal of Control, vol. 19, no. 1, pp. 139-150, 2016.

[34] Y.-T. Wang, Y. Xue, and X. Zhang, "Less conservative robust absolute stability criteria for uncertain neutral-type Lur'e systems with time-varying delays," Journal of the Franklin Institute, vol. 353, no. 4, pp. 816-833, 2016.

[35] Y.-M. Wang, L.-L. Xiong, Y.-K. Li, H.-Y. Zhang, and C. Peng, "Novel stability analysis for uncertain neutral-type Lur'e systems with time-varying delays using new inequality," Mathematical Problems in Engineering, vol. 2017, Article ID 5731325, 13 pages, 2017.

[36] X.-Y. Meng, J. Lam, B.-Z. Du, and H.-J. Gao, “A delay-partitioning approach to the stability analysis of discrete-time systems," Automatica, vol. 46, no. 3, pp. 610-614, 2010.

[37] Z. Yan, H.-J. Gao, J. Lam et al., "Stability and stabilization of delayed T-S fuzzy systems: a delay partitioning approach," IEEE Transactions on Fuzzy Systems, vol. 17, no. 4, pp. 750762, 2009.

[38] O.-M. Kwon, M.-J. Park, J.-H. Park, S.-M. Lee, and E.-J. Cha, "New delay-partitioning approaches to stability criteria for uncertain neutral systems with time-varying delays," Journal of the Franklin Institute, vol. 349, no. 9, pp. 2799-2823, 2012.

[39] P. Mahmoudabadi, M. Shasadeghi, and J. Zarei, "New stability and stabilization conditions for nonlinear systems with timevarying delay based on delay-partitioning approach," ISA Transactions, vol. 70, pp. 46-52, 2017.

[40] L.-D. Guo, X.-H. He, and J.-J. He, "New delay-decomposing approaches to stability criteria for delayed neural networks," Neurocomputing, vol. 189, pp. 123-129, 2016.

[41] P. Park, J.-W. Ko, and C. Jeong, "Reciprocally convex approach to stability of systems with time-varying delays," Automatica, vol. 47, no. 1, pp. 235-238, 2011.

[42] L.-D. Guo, H. Gu, J. Xing, and X.-Q. He, "Asymptotic and exponential stability of uncertain system with interval delay," Applied Mathematics and Computation, vol. 218, no. 19, pp. 9997-10006, 2012.

[43] P.-L. Liu, "Improved delay-range-dependent robust stability for uncertain systems with interval time-varying delay," ISA Transactions, vol. 53, no. 6, pp. 1731-1738, 2014.

[44] K.-B. Shi, H. Zhu, S.-M. Zhong, Y. Zeng, and Y.-P. Zhang, "Improved delay-dependent robust stability criteria for a class of uncertain neutral type Lur'e systems with discrete and distributed delays," Mathematical Problems in Engineering, vol. 2014, Article ID 980351, 14 pages, 2014. 


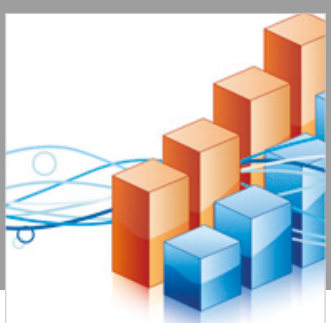

Advances in

Operations Research

\section{-n-m}
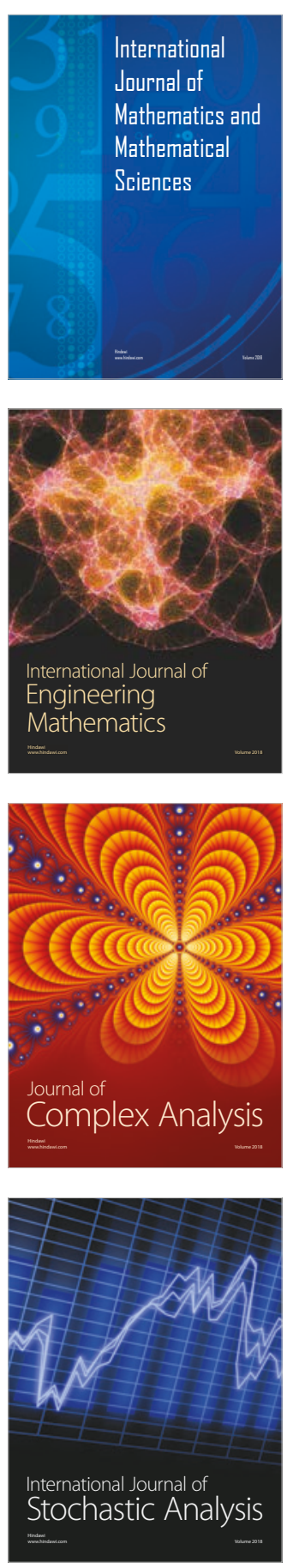
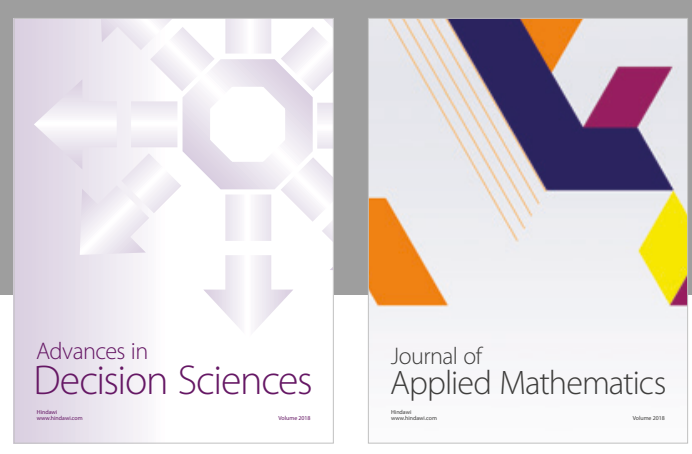

Journal of

Applied Mathematics
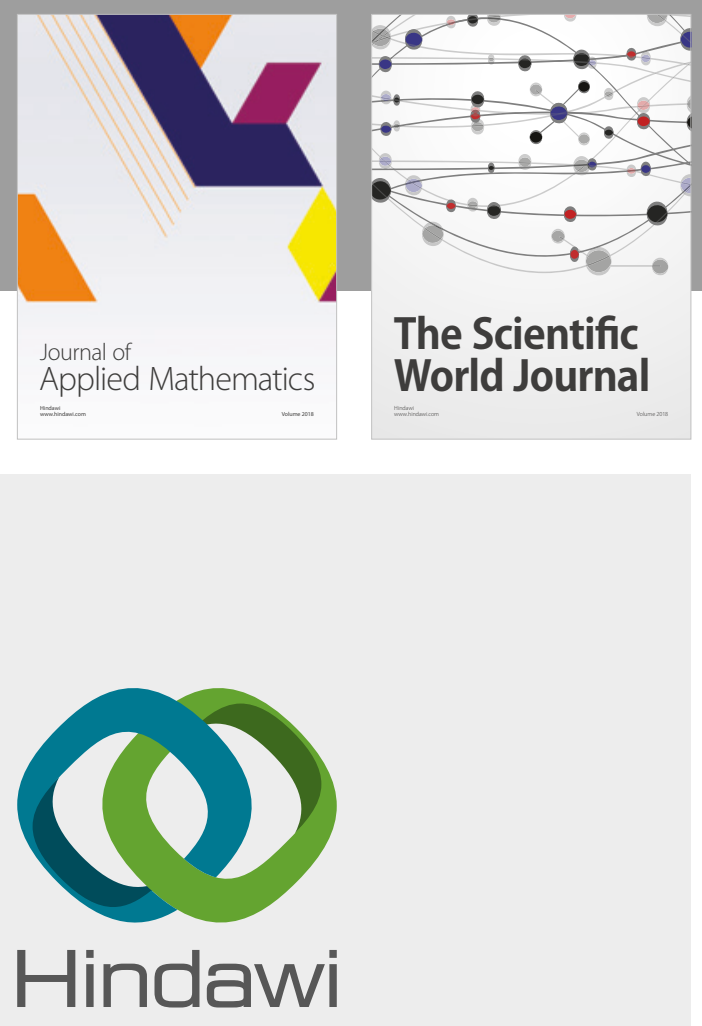

Submit your manuscripts at

www.hindawi.com

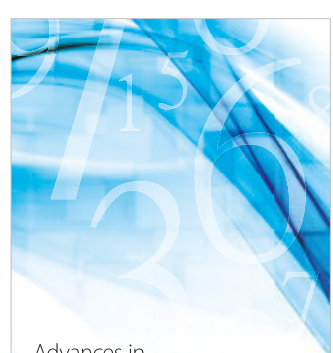

Advances in
Numerical Analysis
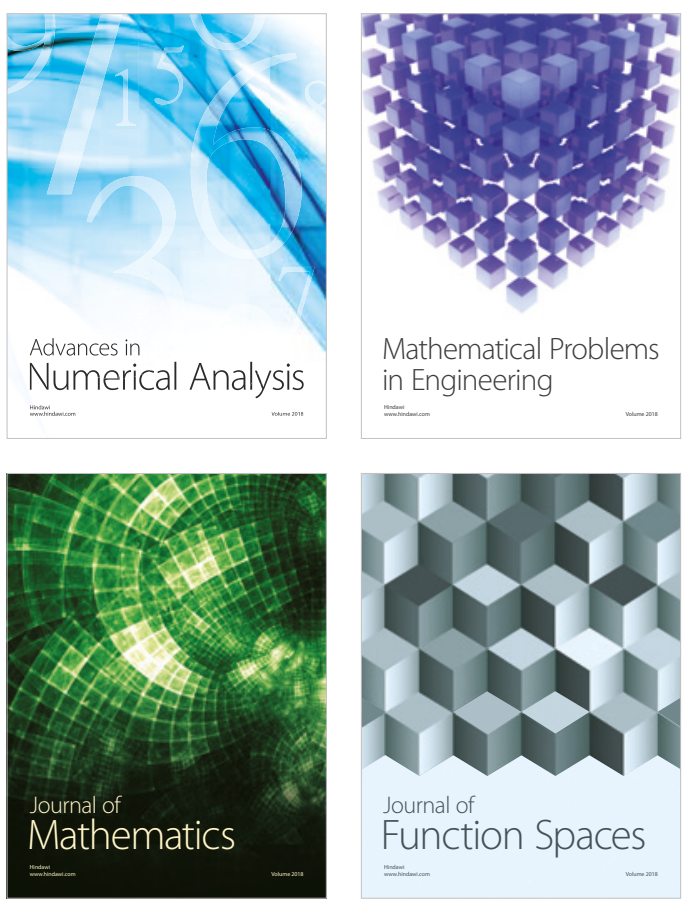

Mathematical Problems in Engineering

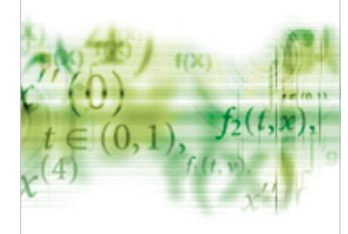

International Journal of

Differential Equations

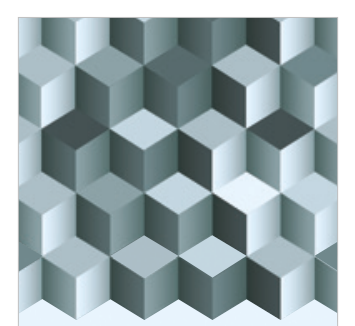

Journal of

Function Spaces

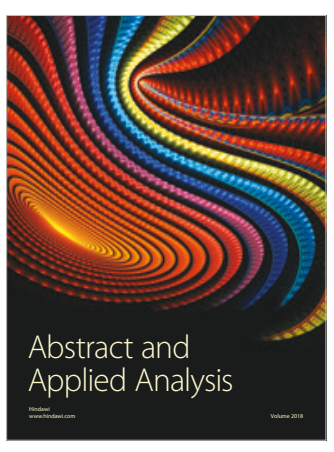

The Scientific

World Journal

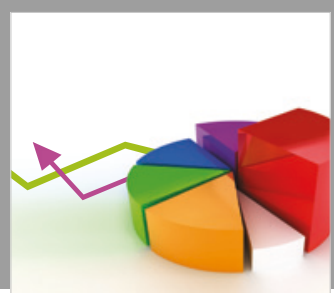

Journal of

Probability and Statistics
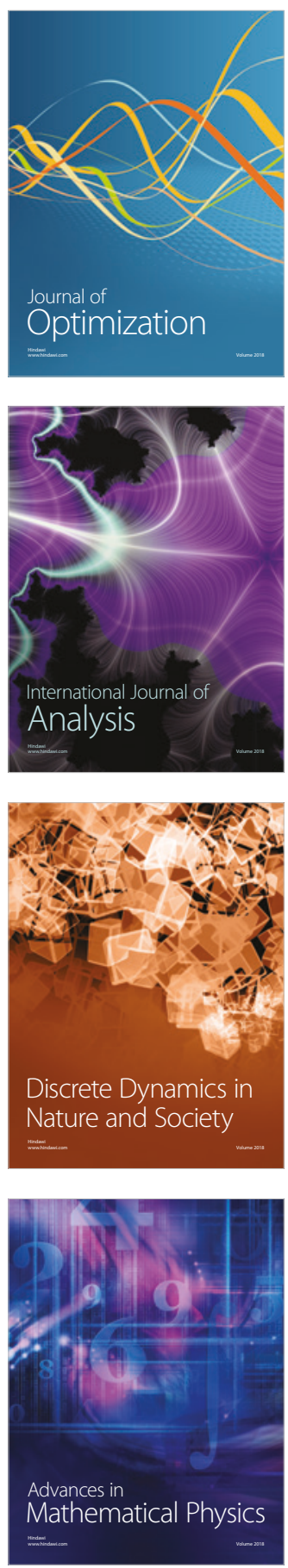